\title{
Loss of HOXD10 expression induced by upregulation of miR-10b accelerates the migration and invasion activities of ovarian cancer cells
}

\author{
IKUE NAKAYAMA $^{1,2}$, MASAHIKO SHIBAZAKI ${ }^{1}$, AKIKO YASHIMA-ABO ${ }^{3}$, FUMIHARU MIURA $^{2}$, \\ TORU SUGIYAMA ${ }^{2}$, TOMOYUKI MASUDA ${ }^{3}$ and CHIHAYA MAESAWA ${ }^{1}$ \\ ${ }^{1}$ Department of Tumor Biology, Institute of Biomedical Science, Departments of ${ }^{2}$ Obstetrics and Gynecology \\ and ${ }^{3}$ Pathology, School of Medicine, Iwate Medical University, Iwate, Japan
}

Received February 22, 2013; Accepted April 5, 2013

DOI: $10.3892 /$ ijo.2013.1935

\begin{abstract}
Small and large non-coding RNAs (ncRNAs) contribute to the acquisition of aggressive tumor behavior in diverse human malignancies. Two types of ncRNAs, miRNA-10b (miR-10b) and homemobox (HOX) transcript antisense RNA (HOTAIR), can suppress the translation of the HOXD10 gene, an mRNA encoding a transcriptional repressor that inhibits the expression of cell migration/invasion-associated genes. Using epithelial ovarian cancer cell lines and primary tumors, we investigated whether miR-10b and/or HOTAIR can regulate the expression of HOXD10, and whether it permits gain of pro-metastatic gene products, matrix metallopeptidase 14 (MMP14) and ras homolog family member C (RHOC). Overexpression of miR-10b induced a decrease in HOXD10 protein expression, and upregulated the migration and invasion abilities in ovarian cancer cell lines $(\mathrm{P}<0.05)$. In these cells, a significant increase of MMP14 and RHOC protein was observed. No significant upregulation of the HOXD10 protein was observed in cells with the treatment of HOTAIR-siRNA. Positive signals for HOXD10 and MMP14 proteins were observed in 47 (69\%) and 25 (37\%) of 68 patients with epithelial ovarian cancers. An inverse correlation between HOXD10 and MMP14 immunoreactivities was observed $(\mathrm{P}<0.05)$, and miR-10b expression was also inversely correlated with HOXD10 protein expression $(\mathrm{P}<0.05)$. These results suggested that downregulation of HOXD10 expression by miR-10b overexpression may induce an increase of pro-metastatic gene products, such as MMP14 and RHOC, and contribute to the acquisition of metastatic phenotypes in epithelial ovarian cancer cells.
\end{abstract}

Correspondence to: Dr Masahiko Shibazaki, Department of Tumor Biology, Institute of Biomedical Science, Iwate Medical University, 2-1-1 Nishitokuta, Yahaba-cho, Iwate 028-3694, Japan

E-mail: mashiba@iwate-med.ac.jp

Key words: ovarian cancer, miRNA-10b, HOXD10, invasion, migration

\section{Introduction}

Ovarian cancer has the highest mortality rate of all gynecologic tumors and represents the fifth leading cause of cancer death for women in the United States. More than 70\% of patients present with disease that has spread beyond the ovaries (1). Ovarian cancer is a highly metastatic disease characterized by intraperitoneal spread $(2,3)$. As disseminated ovarian cancer is usually confined to the surface epithelium within the peritoneal cavity, processes such as cell adhesion, migration, intraperitoneal invasion and proliferation likely play a predominant role in ovarian cancer pathobiology (4). Therefore, the development of more effective treatments for inhibition of invasion/metastasis is urgently needed in patients with advanced ovarian cancer.

Molecular characterization of ovarian cancer makes it possible to develop multiple therapeutic approaches targeting various aspects of the malignancy and to expand the currently available treatment options (5). These include inhibitors of poly (ADP-ribose) polymerase (PARP) (6-8), histone deacetylases (HDACs) (9-11), ERBB family receptors $(12,13)$, heat shock proteins (HSPs) (14), mechanistic target of rapamycin (mTOR)/ hypoxia inducible factor (HIF) $(12,15,16)$ and vascular endothelial growth factor (VEGF) $(8,11,12)$. Some of these approaches might inhibit the migration and invasion of ovarian cancer cells through direct and/or indirect pathways, and their application to management of patients with advanced-stage ovarian cancer is anticipated. However, it has been shown that the efficacy of each agent is limited to a subset of patients exhibiting abnormalities specific to each target molecule. Intensive searches for other candidate molecules involved in the metastatic process are continuing, with the aim of utilizing them for personalized therapy of patients with advanced ovarian cancer $(8,12,17)$.

Small and large non-coding RNAs (ncRNAs) contribute to acquisition of aggressive tumor behavior in a wide range of human malignancies. miR-10b is a particularly interesting candidate given its close correlation with metastatic behavior in human malignancies including breast (18) and gastric cancers (19), renal cell $(20,21)$ and urothelial carcinomas (22), and glioblastoma $(23,24)$. In mammary epithelial cells and breast cancer cells, miR-10b can directly suppress the translation of homeobox D10 (HOXD10), an mRNA encoding a 
transcriptional repressor that inhibits expression of several genes involved in cell migration and extracellular matrix remodeling, such as RHOC and MT1-MMP (MMP14) (18,24,25). Interestingly, HOXD10 is not only targeted by miR-10b, but also by a long ncRNA termed HOX transcript antisense RNA (HOTAIR), which has also been shown to promote breast cancer metastasis. HOTAIR reprograms the chromatin state, causing increased polycomb repressive complex-2 (PRC2) occupancy on promoters of genes that inhibit breast cancer progression, including HOXD10 (26). HOXD10 is a member of the Abd-B homeobox family encoding a protein with a homeobox DNA-binding domain, and its expression is reduced in both breast and endometrial tumors (27). Overexpression of HOXD10 significantly impairs breast tumor cell motility and invasiveness, indicating that HOXD10 may serve as a tumor suppressor $(28,29)$. Although the biological significance of HOXD10 has not been well defined in ovarian cancer, overexpression of MMP14 (30) and RHOC (31), which are negatively regulated by HOXD10, has been observed during progression of the disease. If the miR-10b and/or HOTAIR/HOXD10/MMP14 and/or RHOC axis is involved in the migration/invasion activity of ovarian cancer cells, these would become good candidate target molecules for the development of new personalized therapies for patients with ovarian cancer.

Here, using cell biological methods, we first investigated whether miR-10b and/or HOTAIR could regulate the expression of HOXD10 protein, and thus affect the migration and invasion activities of human ovarian cancer cell lines. We then examined these abnormalities in samples from primary tumors.

\section{Materials and methods}

Cell lines and culture conditions. We used three clear cell adenocarcinoma cell lines (JHOC-5, JHOC-7 and JHOC8), three serous adenocarcinoma cell lines (JHOS-2, JHOS-3 and JHOS-4), one mucinous adenocarcinoma cell line (JHOM-1) and OVCAR-3. They were obtained from Riken Cell Bank (Tsukuba, Japan). All cell lines except OVCAR3 were maintained in DMEM/F12 medium supplemented with 10 or $15 \%$ (JHOS3) fetal bovine serum (FBS), $0.1 \mathrm{mM}$ MEM-non-essential amino acid solution (Life Technologies Inc., Gaithersburg, MD, USA) and $100 \mu \mathrm{l} / \mathrm{ml}$ penicillin-streptomycin. OVCAR3 was maintained in RPMI-1640 (Life Technologies) with $10 \%$ FBS. All tissue culture reagents were obtained from Life Technologies.

Tissue samples. Tissue samples from 68 patients with ovarian cancer and 10 patients with benign ovarian cyst were used for immunohistochemistry and/or real-time quantitative PCR for miR-10b. They were obtained from the Department of Obstetrics and Gynecology, School of Medicine, Iwate Medical University, Morioka, Japan, between 2005 and 2011. The surgical specimens had been fixed in $10 \%$ buffered formalin solution and embedded in paraffin wax. Permission for the study was obtained from the Institutional Review Board (School of Medicine, Iwate Medical University, Iwate, Japan) and written consent had been obtained from all patients before surgery.

Transfection with pre-miRNA precursor and siRNA. Cells were transfected with pre-miR-10b precursor $(50 \mathrm{nM})$ or pre-miR
miRNA precursor negative control using Lipofectamine ${ }^{\mathrm{TM}}$ 2000 (50 nM, Life Technologies) in accordance with the manufacturer's protocol. siRNA oligonucleotides $(50 \mathrm{nM})$ targeting HOTAIR were used as designed by Gupta et al (no. 1 and 2) (26) and predesigned HOTAIR-specific siRNA (no. 3, n272224, Life Technologies) and control non-specific human siRNA (Silencer Select Predesigned siRNA Negative Control no. 2, 4390844, Life Technologies) using Lipofectamine 2000 in accordance with the manufacturer's protocol.

RNA isolation and reverse transcription. Human Ovarian Surface Epithelial Cell total RNA (HOSEipC total RNA) was purchased from ScienCell Research Laboratories (San Diego, CA, USA). Total RNA was extracted from cells using TRIzol reagent in accordance with the manufacturer's protocol (Life Technologies), and transcribed to cDNA with a SuperScript ${ }^{\circledR}$ III First-Strand Synthesis System (Life Technologies). Otherwise, total RNA was extracted from $80-\mu \mathrm{m}$ sections of the formalin-fixed paraffin-embedded material samples (FFPE) using a RecoverAll ${ }^{\mathrm{TM}}$ Total Nucleic Acid Isolation kit (Life Technologies), and reverse-transcribed using a TaqMan microRNA Transcription kit (Life Technologies) and TaqMan Universal PCR Master mix II w/o UNG (Life Technologies) in accordance with the manufacturer's protocol.

Real-time quantitative PCR assay. For quantitative evaluation of the relevant mRNAs, we used Custom TaqMan Gene Expression Assays (HOXD10, Hs00157974_m1; MMP14, Hs01037009_g1; RHOC, Hs00747110_s1; HOTAIR, Hs03296680_s1, Life Technologies) and an ABI PRISM 7500 (Life Technologies). For normalization of the target, glyceraldehydede-3-phosphate dehydrogenase (GAPDH, Life Technologies) was used as an internal control. Triplicate reactions were run per sample, and average fold differences were calculated by normalizing the relative expression $\left(\Delta \Delta \mathrm{C}_{\mathrm{t}}\right.$ values $)$ to the User Bulletin no. 2 (Life Technologies).

miRNA detection. The relative expression levels of miR-10b were measured by a two-step TaqMan assay in accordance with the manufacturer's instructions. Reverse transcription of hsa-miR-10b or the internal control, human U6 were carried out by a TaqMan microRNA reverse transcription kit (Life Technologies). Then, real-time PCR reactions were performed using standard TaqMan ${ }^{\circledR}$ PCR reagents and TaqMan ${ }^{\circledR}$ MicroRNA assays for hsa-miR-10b and U6 (Life Technologies). The expression of miR-10b relative to U6 was determined using the $\Delta \Delta \mathrm{C}_{\mathrm{t}}$ method.

Western blot analysis. Seventy-two hours after transfection, nucleic and cytoplasmic proteins were collected using NE-PER $^{\mathrm{TM}}$ Nuclear and Cytoplasmic Extraction Reagent (Pierce, Thermo Fisher Scientific Inc., Rockford, IL, USA). Equal amounts of protein sample were separated by $4-12 \%$ $\mathrm{Nu}-\mathrm{PAGE}$ and transferred to PVDF membranes by electroblotting. The primary antibodies used were anti-HOXD10 (ABE128; Millipore, Billerica, MA) and anti-MMP14 (ab3644; Abcam, Cambridge, MA, USA), both diluted in immunoreaction enhancer solution (Can Get Signal Solution1, Toyobo, Osaka, Japan). We also used primary antibodies against RhoC (\#3430; Cell Signaling, Beverly, MA, USA), GAPDH (Clone 
1D4; Covance, Princeton, NJ, USA) and lamin B (sc-6217; Santa Cruz Biotechnology, Santa Cruz, CA, USA). Signals were detected using an ECL Prime Western Blotting Detection kit (GE Healthcare, Buckinghamshire, UK) and ChemidDoc XRS (Bio-Rad Laboratories, Hercules, CA, USA). The intensity of the detected signals was measured by using Image $\mathbf{J}$ (freely available java-based public-domain image processing and analysis program developed at the National Institutes of Health).

In vitro migration and invasion assays. For Transwell migration assays, $2.5-5 \times 10^{4}$ cells were placed in the top chamber on a non-coated membrane (24-well insert; pore size, $8 \mu \mathrm{m}$; BD Bioscience, San Jose, CA, USA). For invasion assays, $2.5-5 \times 10^{4}$ cells were placed in the top chamber on a Matrigel-coated membrane (24-well insert; pore size, $8 \mu \mathrm{m}$; BD Bioscience). In both assays, the cells were placed in medium containing $1 \% \mathrm{FBS}$, and medium supplemented with $20 \%$ FBS was used as a chemoattractant in the lower chamber. The cells were incubated for $72 \mathrm{~h}$, and those that did not migrate or invade through the pores were removed with a cotton swab. Cells were fixed with methanol, stained with DAPI (Dojindo Laboratories, Kumamoto, Japan) and counted. Individual experiments had triplicate inserts, and five randomly selected fields were counted per insert.

Immunohistochemistry. Four-micrometer-thick sections were cut from formalin-fixed, paraffin-embedded samples, and stained with hematoxylin and eosin. Serial sections were stained using the avidin-biotin system and antigen retrieval methods on a Ventana automated immunostainer with the Ventana immunohistochemistry detection system (Ventana Medical Systems, Tucson, AZ, USA), in accordance with the manufacturer's manual. The primary antibody used for MMP14 immunostaining was a rabbit polyclonal anti-MMP14 antibody (Abcam), and for HOXD10 immunostaining a mouse monoclonal anti-HOXD10 antibody (Santa Cruz Biotechnology). Quantitative comparative analysis of immunohistochemical staining was carried out in each case. Two independent pathologists performed quantitative assessment of immunohistochemical staining. For HOXD10, staining in nuclei was graded as follows: 0 , no immunoreactive cells evident; 1 , proportion of immunoreactive cells $<20 \% ; 2,20-70 \% ; 3,>70 \%$. For the final estimation of HOXD10 immunoreactivity, patients with a score of $0 / 1$ were considered negative, and those with a score of $2 / 3$ as positive. MMP14 imunoreactivity was graded according to the number of immunoreactive cells (proportion score) and staining intensity (intensity score). In brief, proportion scores were graded as follows: 0 , no immunoreactive cells evident; $1,<20 \% ; 2,20-70 \% ; 3,>70 \%$. Intensity scores were graded as follows: 0 , no immunoreactivity; 1 , staining intensity weak; 2 , intermediate; and 3, strong. The finally estimated immunoreactivity was considered negative if the sum of the proportion and intensity scores ranged from 0 to 3 and positive if the sum ranged from 4 to 6 .

Statistical analysis. Data were analyzed by the Mann-Whitney $\mathrm{U}$ test for non-parametric samples or Fisher's exact test. Differences at $\mathrm{p}<0.05$ were considered to be statistically significant.
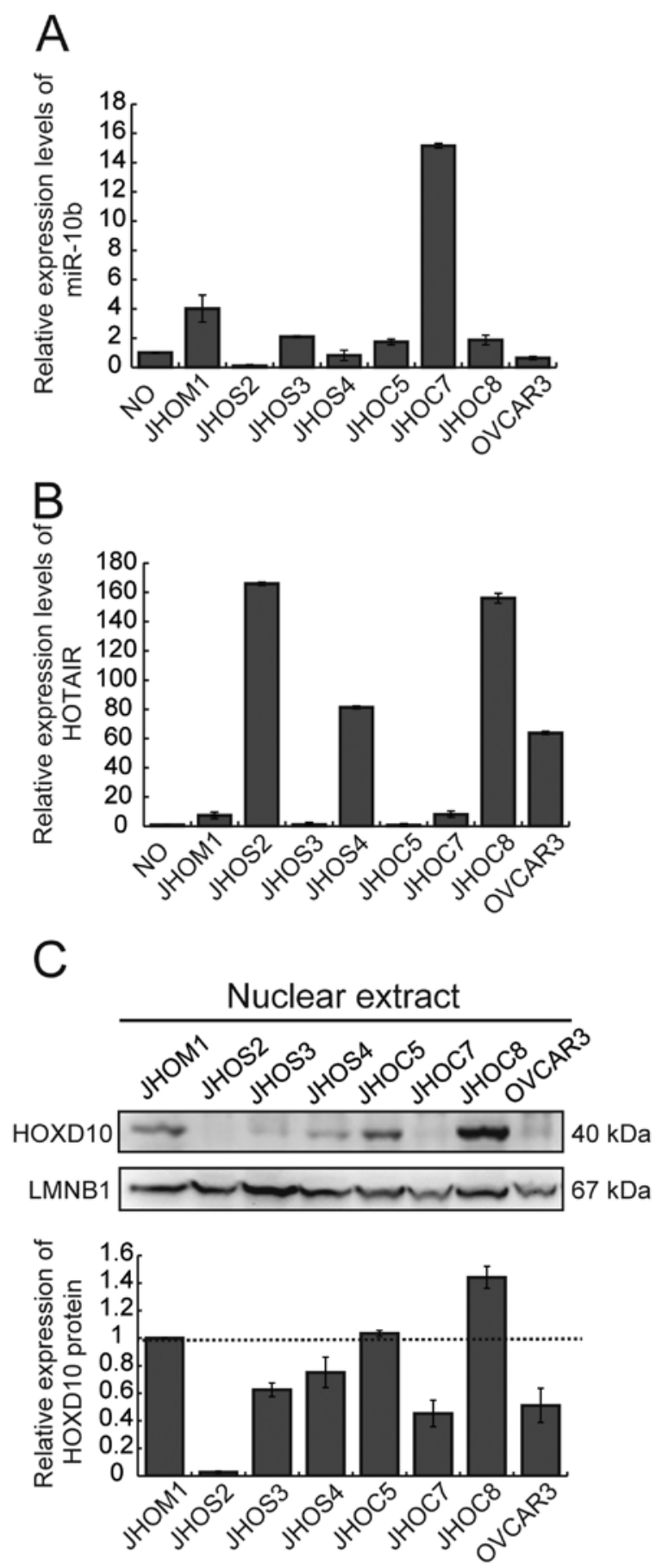

Figure 1. Quantitative results for miR-10b, HOTAIR and HOXD10 protein expression in epithelial ovarian cancer cell lines and/or normal ovary. Relative expression of (A) miR-10b and (B) HOTAIR in comparison with RNA extracted from normal ovary (NO) was evaluated by real-time quantitative PCR. (C) Quantification of HOXD10 protein expression using western blot analysis is presented as a ratio relative to the expression level of JHOM1.

\section{Results}

Expression of miR-10b, HOTAIR and HOXD10 protein in ovarian cancer cell lines. We first examined the expression of miR-10b and HOTAIR in 8 ovarian cancer cell lines and RNAs extracted from human normal ovary (Fig. 1A and B). The expression level of both ncRNAs varied among the cell lines. Gain of miR-10b was observed in 2 cell lines (JHOM1 

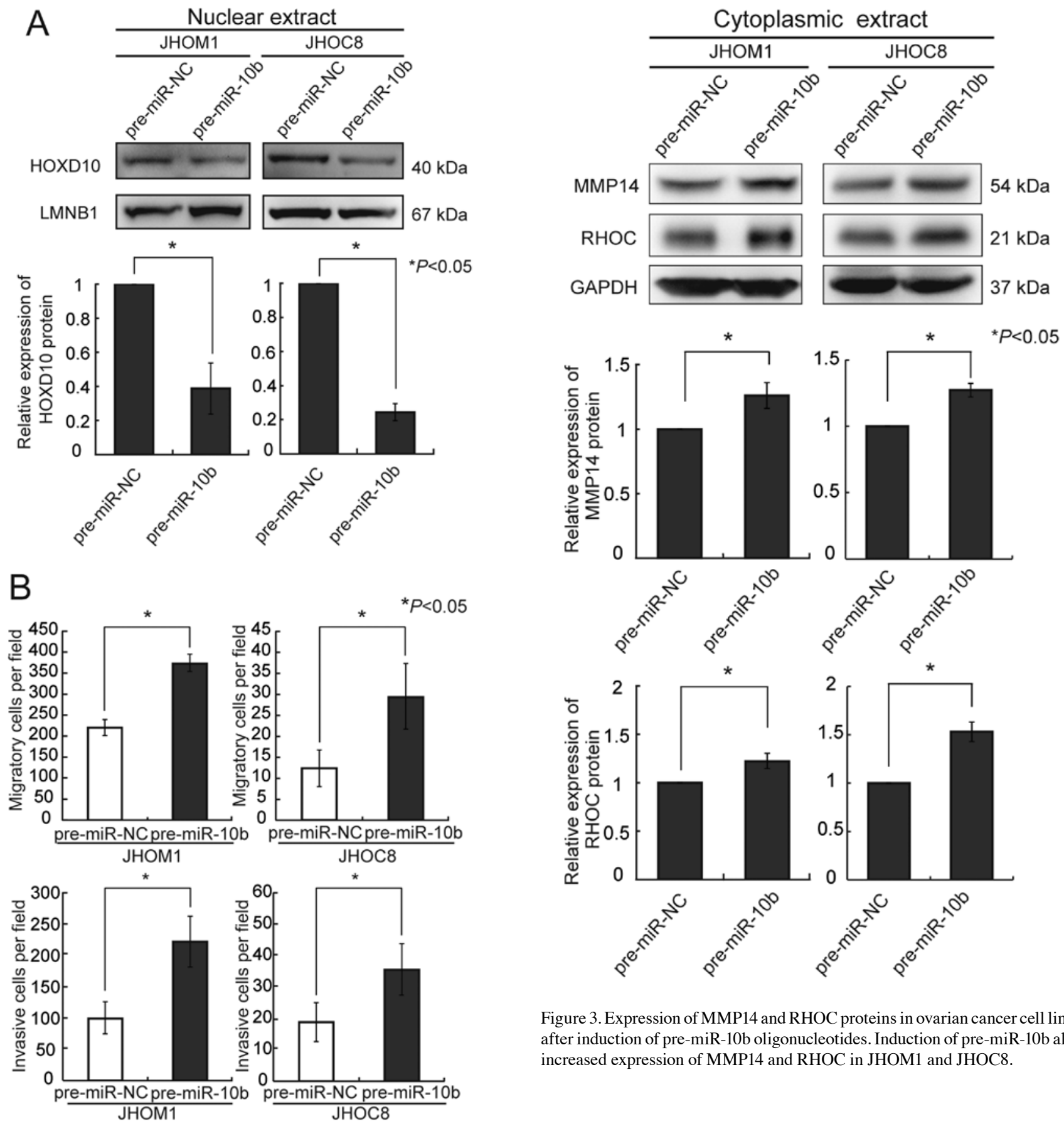

Figure 2. Expression of HOXD10 protein and results of in vitro migration and invasion assays of ovarian cancer cell lines after induction of pre-miR-10b oligonucleotides. (A) MiR-10b was overexpressed by introducing pre-miR-10b oligonucleotides into JHOM1 and JHOC8, and it decreased the expression of HOXD10 in both cell lines. (B) Induction of pre-miR-10b significantly increased the migration/invasion activity of JHOM1 and JHOC8.

and JHOC7) in comparison with normal ovary (Fig. 1A). Overexpression of HOTAIR was observed in 4 cell lines (JHOS2, JHOS4, JHOC8 and OVCAR3) in comparison with normal ovary (Fig. 1B). We examined the correlation between the expression level of miR-10b and/or HOTAIR, and HOXD10 protein (Fig. 1C) in these cell lines, but no significant relationship between the expression of ncRNAs and HOXD10 protein was evident.

Next, using a cell biological approach, we investigated whether ovarian cancers showed an inverse correlation between the expression of miR-10b and/or HOTAIR, and HOXD10 protein, which has been proven previously in other tumors.

Overexpression of precursor miR-10b in ovarian cancer cell lines. Overexpression of precursor miR-10b induced a decrease of HOXD10 protein in the ovarian cancer cell lines JHOM1 and JHOC8 (Fig. 2A). We also examined cytoplasmic extracts for expression of MMP14 and RHOC protein. A significant increase of both proteins was observed in each cell line (Fig. 3). In these cells, both migration and invasion activities were markedly upregulated $72 \mathrm{~h}$ after transfection, in comparison with the controls (Fig. 2B).

We used a locked nucleic acid (LNA; Exiqon, Vedbaek, Denmark) approach to knockdown miR-10b in cells (JHOM1 


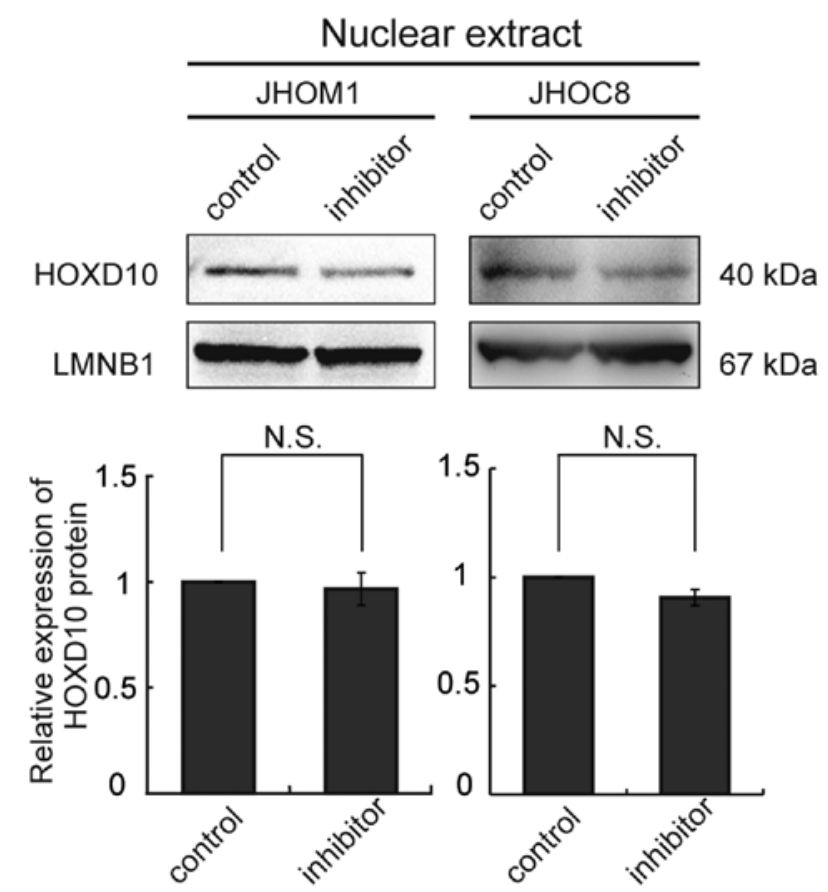

Figure 4. Relative expression levels of HOXD10 protein in two ovarian cancer cell lines transfected with miRCURY LNA inhibitor targeting miR-10b. HOXD10 protein levels did not increase after transfection with miRCURY LNA inhibitor targeting miR-10b.

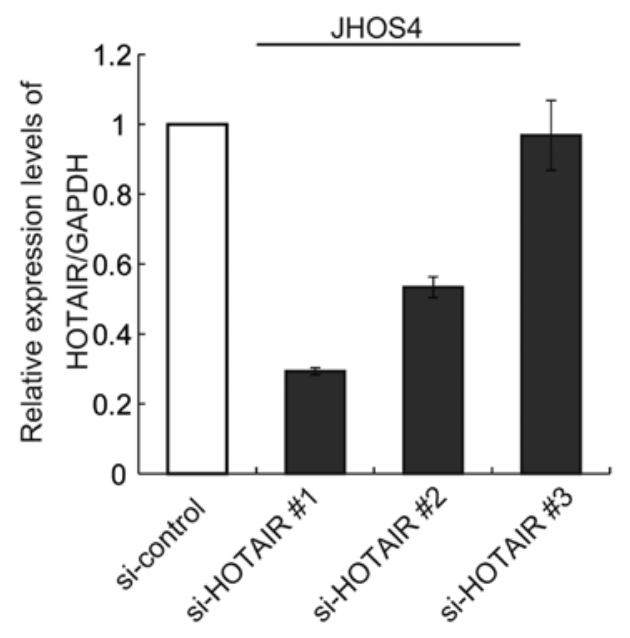

Figure 5. Relative levels of HOTAIR in ovarian cancer cell lines after transfection with siRNA targeting HOTAIR (three individual duplexes). Only si-HOTAIR no. 1 was able to achieve significant downregulation of HOTAIR expression by $80 \%$ in comparison with the control siRNA in JHOS 4 cells.

and JHOC8) showing relative overexpression of miR-10b, but this failed to upregulate HOXD10 protein expression in these cell lines (Fig. 4).

Knockdown of HOTAIR in ovarian cancer cell lines. Next, we tested the knockdown efficiency of HOTAIR expression using siRNAs (no. 1, 2 and 3). Only si-HOTAIR no. 1 was able to achieve significant downregulation of HOTAIR expression by $80 \%$ in comparison with the control siRNAin JHOS 4 cells (Fig. 5). We then treated JHOS4 and OVCAR3, which express
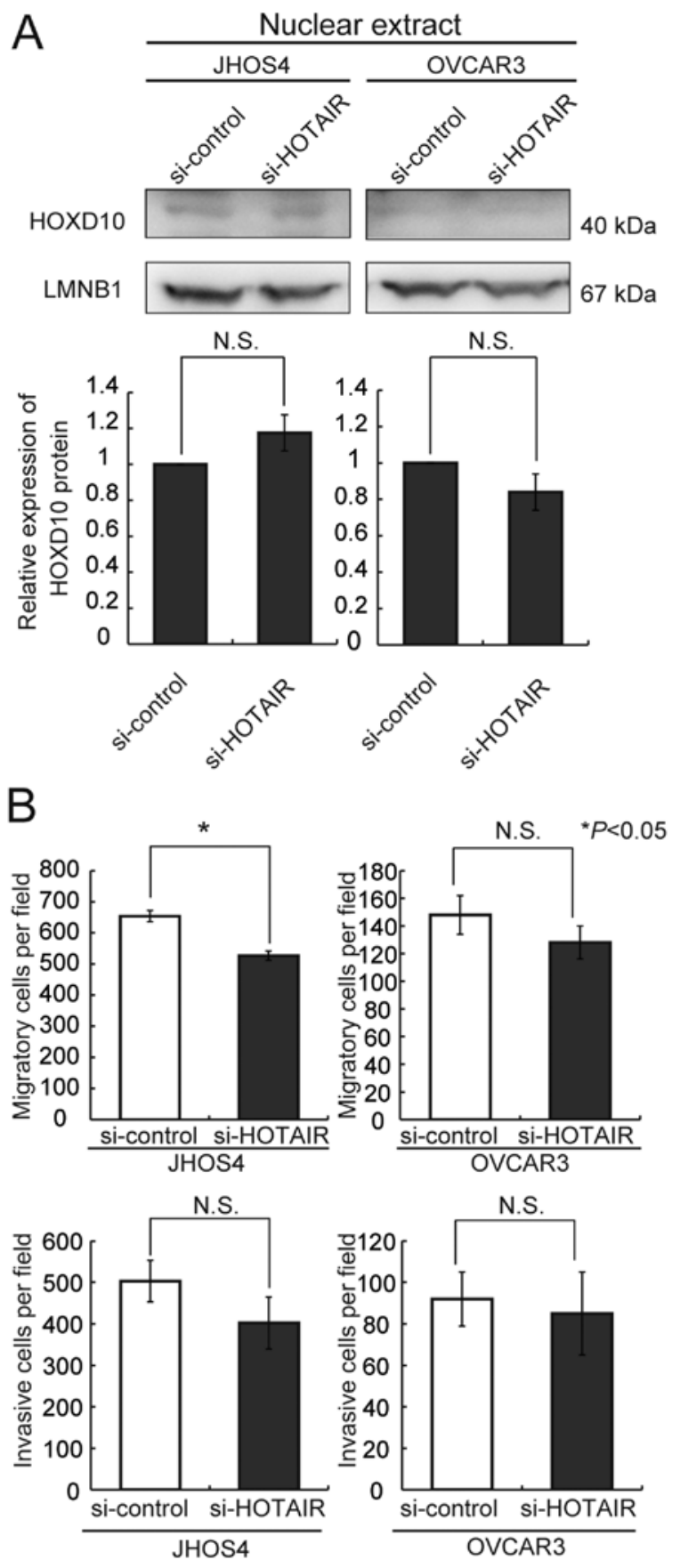

Figure 6. Expression of HOXD10 protein and results of in vitro migration and invasion assays of ovarian cancer cell lines after knockdown of HOTAIR. (A) The siRNA treatment did not alter HOXD10 expression in either ovarian cancer cell line (showing relative overexpression of HOXD10). (B) Knockdown of HOTAIR significantly induced a decrease of cell migration activity of JHOS4. Although a tendency for a decrease of migration and/or invasion activities was found in both cell lines, it was not statistically significant.

high levels of HOTAIR, with si-HOTAIR no. 1. No significant upregulation of HOXD10 protein expression was observed in either of the cell lines after treatment with HOTAIR siRNA (Fig. 6A). But, knockdown of HOTAIR was significantly induced decreasing of only cell migration activity of JHOS4 (Fig. 6B). Although both cell lines showed a tendency for decreased migration and/or invasion activities, the difference was not significant relative to that achieved with control siRNA 

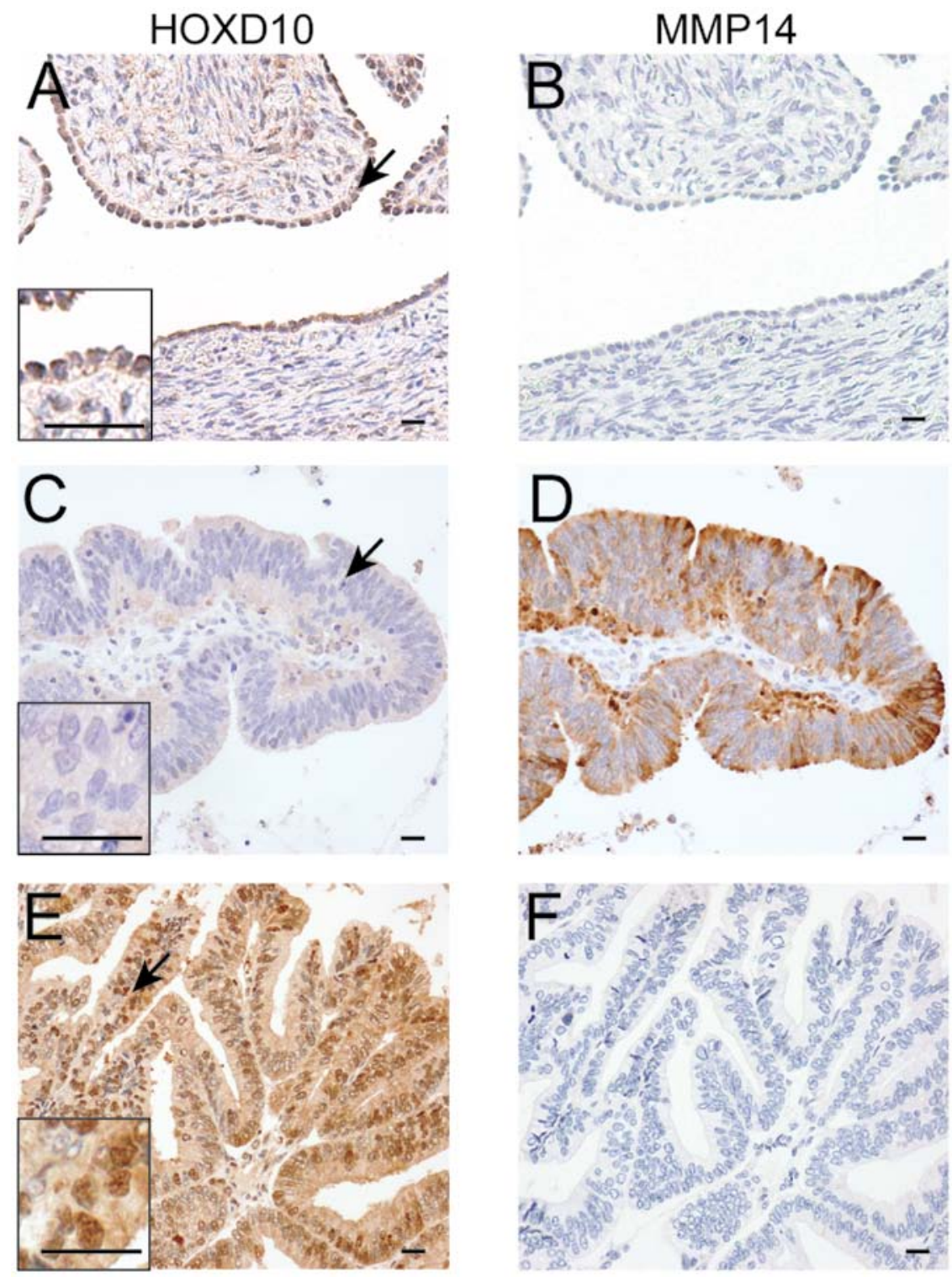

Figure 7. Immunohistochemistry for HOXD10 and MMP14 proteins in normal ovary and ovarian cancers. Positive immunoreactivity for HOXD10 protein was observed (A) in the nuclei of normal ovary mesothelial surface lining cells, whereas MMP14 was negative (B). The immunoreactivities of HOXD10 and MMP14 (C-F) were inversely correlated in the two ovarian cancers. Scale bar, $20 \mu \mathrm{m}$.

(Fig. 6B). Therefore, we concluded that the decrease of the migration and invasion activities resulting from treatment with HOTAIR siRNA might not be attributable to alterations of HOXD10 protein.

Immunohistochemistry for HOXD10 and MMP14 proteins and its relationship with miR-10b expression. We next investigated miR-10b expression in primary ovarian cancers. As in vitro data had suggested that upregulation of miR-10b expression had induced a decrease of HOXD10 protein expression followed by overexpression of MMP14, we immunohistochemically examined the expressions of HOXD10 and MMP14, and also miR-10b, using real-time quantitative PCR in 68 patients with primary ovarian cancer.

In normal ovaries, positive immunoreactivity for HOXD10 protein was observed in the nuclei of mesothelial surface lining cells, while MMP14 was negative (Fig. 7A and B). Positive
Table I. Immunohistochemistry for HOXD10 and MMP14 in 68 ovarian cancers.

\begin{tabular}{lrrr}
\hline & \multicolumn{2}{c}{ MMP14 } & \\
\cline { 2 - 3 } & Positive & Negative & P-value \\
\hline HOXD10 & & & \\
Positive & 12 & 35 & 0.005 \\
Negative & 13 & 8 & \\
\hline
\end{tabular}

signals for HOXD10 and MMP14 proteins were observed in $47(69 \%)$ and $25(37 \%)$ of 68 patients. A significant inverse correlation between immunoreactivity of HOXD10 and MMP14 was observed (Fig. 7C-F, Table I). We also examined the correlations between HOXD10 and/or MMP14 immunore- 
Table II. Correlation between HOXD10 or MMP14 immunoreactivity and clinicopathological factors in 68 patients with ovarian cancer.

\begin{tabular}{|c|c|c|c|c|c|c|}
\hline \multirow[b]{2}{*}{ Factor } & \multicolumn{3}{|c|}{ HOXD10 } & \multicolumn{3}{|c|}{ MMP14 } \\
\hline & $\begin{array}{c}\text { Positive } \\
(\mathrm{n}=47)\end{array}$ & $\begin{array}{c}\text { Negative } \\
(n=21)\end{array}$ & P-value & $\begin{array}{c}\text { Positive } \\
(\mathrm{n}=25)\end{array}$ & $\begin{array}{c}\text { Negative } \\
(\mathrm{n}=43)\end{array}$ & P-value \\
\hline Age & $\begin{array}{c}53.6 \\
(25-80)\end{array}$ & $\begin{array}{c}55 \\
(34-80)\end{array}$ & & $\begin{array}{c}52.32 \\
(34-80)\end{array}$ & $\begin{array}{c}55.18 \\
(25-80)\end{array}$ & \\
\hline Stage & & & & & & \\
\hline $\begin{array}{l}\text { I } \\
\text { Other }\end{array}$ & $\begin{array}{l}32 \\
15\end{array}$ & $\begin{array}{r}12 \\
9\end{array}$ & 0.353 & $\begin{array}{r}18 \\
7\end{array}$ & $\begin{array}{l}26 \\
19\end{array}$ & 0.244 \\
\hline Histology & & & & & & \\
\hline $\begin{array}{l}\text { Serous } \\
\text { Other }\end{array}$ & $\begin{array}{l}12 \\
35\end{array}$ & $\begin{array}{r}9 \\
12\end{array}$ & 0.955 & $\begin{array}{r}9 \\
16\end{array}$ & $\begin{array}{l}12 \\
31\end{array}$ & 0.592 \\
\hline Node status & & & & & & \\
\hline $\begin{array}{l}\text { Positive } \\
\text { Negative } \\
\text { Not done }\end{array}$ & $\begin{array}{r}6 \\
36 \\
5\end{array}$ & $\begin{array}{r}3 \\
17 \\
1\end{array}$ & 0.557 & $\begin{array}{r}3 \\
21 \\
1\end{array}$ & $\begin{array}{r}6 \\
32 \\
5\end{array}$ & 0.647 \\
\hline
\end{tabular}

A

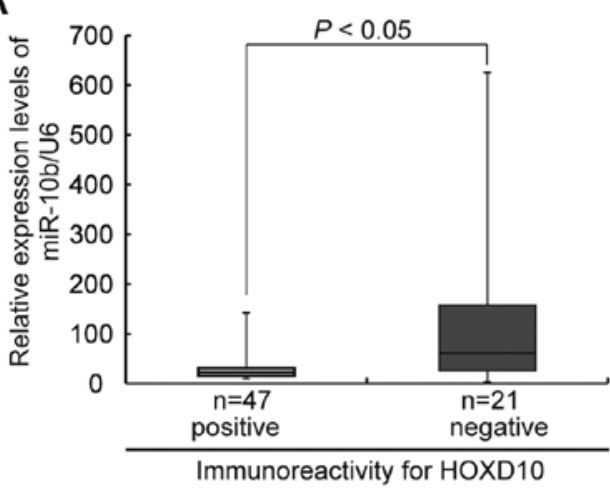

B

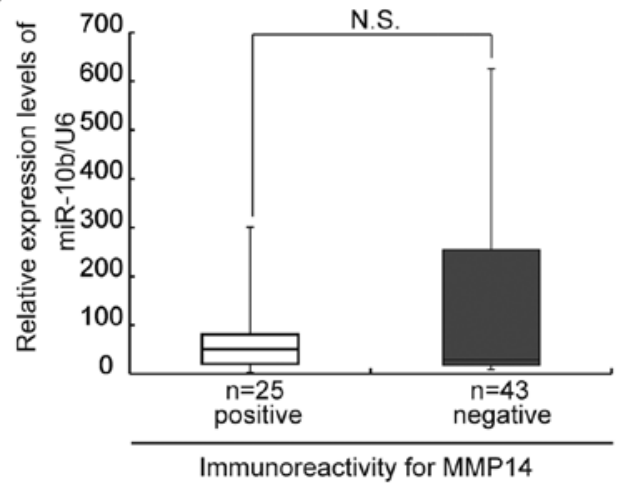

Figure 8. Relationship between miR-10b expression and immunohistochemical expression of HOXD10 and MMP14 in 68 ovarian cancers. (A) An inverse correlation between miR-10b and HOXD10 immunoreactivity was observed, (B) but no such correlation was evident for MMP14.

activity and clinicopathological parameters in the patients, but no significant relationship was found (Table II). However, as $48(70 \%)$ of the 68 studied patients had stage I/II cancer, and various histological subtypes of ovarian cancer were included, some bias was probably present, making them unsuitable for statistical evaluation. The expression level of miR-10b was inversely correlated with HOXD10 protein expression (Fig. 8A), but not with MMP14 expression (Fig. 8B).

\section{Discussion}

Many studies have investigated the association between miRNA alterations and the biological characteristics of ovarian cancer, and several candidate miRNAs have been nominated (32-39). These miRNAs hold promise for the detection of earlystage ovarian cancer, evaluation of prognosis/drug resistance, and development of targeted cancer treatment. In vitro and in vivo studies have suggested that miR-7 (34,38), miR-21 (38),
miR-34 (40,41), miR-22 (39), and miR-429 (members of the miR-200 family) (37) may directly and/or indirectly control the expression of metastasis-associated genes related to invasion/ migration and epithelial-mesenchymal transition (EMT). The present study newly highlighted miR-10b as a novel inducer of metastasis in ovarian cancers.

As mentioned in the introduction, miR-10b is a strong inducer of metastasis in breast cancer cells (18), and is also one of the most upregulated miRNAs in human pancreatic adenocarcinomas $(42,43)$ and glioblastomas $(24,44-46)$, which are both highly metastatic and/or invasive cancers. miR-10b suppresses the synthesis of the HOXD10 protein, permitting expression of the pro-metastatic gene products RHOC urokinase plasminogen activator receptor (uPAR), $\alpha 3$-integrin, and MMP14 $(18,25)$. In addition, TWIST, a wel-known transcriptional factor related to EMT, activates the transcription of miR-10b by binding directly to an E-box sequence proximal to its putative promoter (18). Although miR-10b does not trigger EMT by itself, it might be 
required for TWIST-induced cell motility and invasiveness in ovarian cancer cells (18).

Evidence for a significant role of HOTAIR in metastasis of several malignancies has been increasing. Since Gupta et al (26) first documented that loss of HOTAIR can inhibit the invasiveness of cancer cells, particularly those possessing excessive PRC2 activity, similar evidence has been detected not only in breast cancer (47) but also gastrointestinal stromal tumors (48), and colorectal (49) and hepatocellular carcinomas (50). HOTAIR reprograms the chromatin state, causing increased PRC2 occupancy on promoters of genes, including HOXD10 (26), inhibiting breast cancer progression. In the present study, although we failed to demonstrate that HOTAIR can repress HOXD10 expression in ovarian cancer, and did not examine the promoter status of HOXD10, knockdown of HOTAIR appeared to decrease the migration/invasion activity of ovarian cancer cells (Fig. 6B). The effect of HOTAIR on invasion and migration might be exerted via pathways other than HOXD10, such as MMP9 and VEGF (50).

Targeting of metastasis-promoting miRNAs is emerging as a novel therapeutic strategy for cancer treatment. The effect of the miR-10b antagomir has been reported in a 4T1 mouse mammary tumor metastasis model $(51,52)$. Administration of miR-10b antagomirs to mice bearing highly metastatic cells does not reduce primary mammary tumor growth, but blocks dissemination of cancer cells from the primary tumor (52).

Because the miR-10b antagomir would prevent metastatic intraperitoneal dissemination, it would be worth investigating whether it can be added as a prophylactic therapy for patients with early-stage ovarian cancer. Moreover, further studies using clinical samples would be warranted to clarify whether quantification of miR-10b in intraperitoneal fluid and/or peripheral blood would be applicable as a biomarker of intraperitoneal dissemination of ovarian cancers.

\section{Acknowledgements}

This study was supported in part by Grants-in-Aid for Scientific Research (22390071), the MIAST project, and a Grant-in-Aid for Strategic Medical Science Research from the Ministry of Education, Culture, Sports, Science and Technology of Japan.

\section{References}

1. Siegel R, Naishadham D and Jemal A: Cancer statistics, 2012. CA Cancer J Clin 62: 10-29, 2012.

2. Hu X, Li D, Zhang W, Zhou J, Tang B and Li L: Matrix metalloproteinase-9 expression correlates with prognosis and involved in ovarian cancer cell invasion. Arch Gynecol Obstet 286: $1537-1543,2012$.

3. Fidler IJ: The pathogenesis of cancer metastasis: the 'seed and soil' hypothesis revisited. Nat Rev Cancer 3: 453-458, 2003.

4. Fishman DA, Liu Y, Ellerbroek SM and Stack MS: Lysophosphatidic acid promotes matrix metalloproteinase (MMP) activation and MMP-dependent invasion in ovarian cancer cells. Cancer Res 61: 3194-3199, 2001.

5. Sourbier C: Ovarian cancer: emerging molecular-targeted therapies. Biologics 6: 147-154, 2012.

6. Ledermann J, Harter P, Gourley C, et al: Olaparib maintenance therapy in platinum-sensitive relapsed ovarian cancer. N Engl J Med 366: 1382-1392, 2012.

7. Yamamoto N, Nokihara H, Yamada Y, et al: A phase I, dose-finding and pharmacokinetic study of olaparib (AZD2281) in Japanese patients with advanced solid tumors. Cancer Sci 103: 504-509, 2012
8. Campos SM and Ghosh S: A current review of targeted therapeutics for ovarian cancer. J Oncol 2010: 149362, 2010.

9. Kumagai T, Wakimoto N, Yin D, et al: Histone deacetylase inhibitor, suberoylanilide hydroxamic acid (Vorinostat, SAHA) profoundly inhibits the growth of human pancreatic cancer cells. Int J Cancer 121: 656-665, 2007.

10. Ramalingam SS, Parise RA, Ramanathan RK, et al: Phase I and pharmacokinetic study of vorinostat, a histone deacetylase inhibitor, in combination with carboplatin and paclitaxel for advanced solid malignancies. Clin Cancer Res 13: 3605-3610, 2007.

11. Itamochi H: Targeted therapies in epithelial ovarian cancer: molecular mechanisms of action. World J Biol Chem 1: 209-220, 2010.

12. Yap TA, Carden CP and Kaye SB: Beyond chemotherapy: targeted therapies in ovarian cancer. Nature reviews. Cancer 9: 167-181, 2009.

13. Pautier P, Joly F, Kerbrat P, et al: Phase II study of gefitinib in combination with paclitaxel (P) and carboplatin (C) as second-line therapy for ovarian, tubal or peritoneal adenocarcinoma (1839IL/0074). Gynecol Oncol 116: 157-162, 2010.

14. Cohen M, Dromard M and Petignat P: Heat shock proteins in ovarian cancer: a potential target for therapy. Gynecol Oncol 119: 164-166, 2010.

15. Behbakht K, Sill MW, Darcy KM, et al: Phase II trial of the mTOR inhibitor, temsirolimus and evaluation of circulating tumor cells and tumor biomarkers in persistent and recurrent epithelial ovarian and primary peritoneal malignancies: a Gynecologic Oncology Group study. Gynecol Oncol 123: 19-26, 2011.

16. Huynh H, Teo CC and Soo KC: Bevacizumab and rapamycin inhibit tumor growth in peritoneal model of human ovarian cancer. Mol Cancer Ther 6: 2959-2966, 2007.

17. Lord CJ and Ashworth A: Targeted therapy for cancer using PARP inhibitors. Curr Opin Pharmacol 8: 363-369, 2008.

18. Ma L, Teruya-Feldstein J and Weinberg RA: Tumour invasion and metastasis initiated by microRNA-10b in breast cancer. Nature 449: 682-688, 2007.

19. Liu Z, Zhu J, Cao H, Ren H and Fang X: miR-10b promotes cell invasion through RhoC-AKT signaling pathway by targeting HOXD10 in gastric cancer. Int J Oncol 40: 1553-1560, 2012.

20. Wotschofsky Z, Liep J, Meyer HA, et al: Identification of metastamirs as metastasis-associated microRNAs in clear cell renal cell carcinomas. Int J Biol Sci 8: 1363-1374, 2012.

21. White NM, Khella HW, Grigull J, et al: miRNA profiling in metastatic renal cell carcinoma reveals a tumour-suppressor effect for miR-215. Br J Cancer 105: 1741-1749, 2011.

22. Zaravinos A, Radojicic J, Lambrou GI, et al: Expression of miRNAs involved in angiogenesis, tumor cell proliferation, tumor suppressor inhibition, epithelial-mesenchymal transition and activation of metastasis in bladder cancer. J Urol 188: 615-623, 2012.

23. Karsy M, Arslan E and Moy F: Current progress on understanding microRNAs in glioblastoma multiforme. Genes Cancer 3: 3-15, 2012.

24. Sasayama T, Nishihara M, Kondoh T, Hosoda K and Kohmura E: MicroRNA-10b is overexpressed in malignant glioma and associated with tumor invasive factors, uPAR and RhoC. Int J Cancer 125: 1407-1413, 2009.

25. Myers C, Charboneau A, Cheung I, Hanks D and Boudreau N: Sustained expression of homeobox D10 inhibits angiogenesis. Am J Pathol 161: 2099-2109, 2002.

26. Gupta RA, Shah N, Wang KC, et al: Long non-coding RNA HOTAIR reprograms chromatin state to promote cancer metastasis. Nature 464: 1071-1076, 2010.

27. Osborne J, Hu C, Hawley C, Underwood LJ, O'Brien TJ and Baker VV: Expression of HOXD10 gene in normal endometrium and endometrial adenocarcinoma. J Soc Gynecol Investig 5: 277-280, 1998.

28. Reddy SD, Ohshiro K, Rayala SK and Kumar R: MicroRNA-7, a homeobox D10 target, inhibits p21-activated kinase 1 and regulates its functions. Cancer Res 68: 8195-8200, 2008.

29. Carrio M, Arderiu G, Myers C and Boudreau NJ: Homeobox D10 induces phenotypic reversion of breast tumor cells in a threedimensional culture model. Cancer Res 65: 7177-7185, 2005.

30. Roy R, Yang J and Moses MA: Matrix metalloproteinases as novel biomarkers and potential therapeutic targets in human cancer. J Clin Oncol 27: 5287-5297, 2009.

31. Horiuchi A, Imai T, Wang C, et al: Up-regulation of small GTPases, RhoA and RhoC, is associated with tumor progression in ovarian carcinoma. Lab Invest 83: 861-870, 2003. 
32. Zaman MS, Maher DM, Khan S, Jaggi M and Chauhan SC: Current status and implications of microRNAs in ovarian cancer diagnosis and therapy. J Ovarian Res 5: 44, 2012.

33. Chang S and Sharan SK: BRCA1 and MicroRNAs: emerging networks and potential therapeutic targets. Mol Cells 34: 425-432, 2012

34. Bovicelli A, D'Andrilli G and Giordano A: New players in ovarian cancer. J Cell Physiol 226: 2500-2504, 2011.

35. Asadollahi R, Hyde CA and Zhong XY: Epigenetics of ovarian cancer: from the lab to the clinic. Gynecol Oncol 118: 81-87, 2010.

36. Shahab SW, Matyunina LV, Hill CG, et al: The effects of MicroRNA transfections on global patterns of gene expression in ovarian cancer cells are functionally coordinated. BMC Med Genomics 5: 33, 2012.

37. Chen J, Wang L, Matyunina LV, Hill CG and McDonald JF: Overexpression of miR-429 induces mesenchymal-to-epithelial transition (MET) in metastatic ovarian cancer cells. Gynecol Oncol 121: 200-205, 2011.

38. Lou Y, Yang X, Wang F, Cui Z and Huang Y: MicroRNA-21 promotes the cell proliferation, invasion and migration abilities in ovarian epithelial carcinomas through inhibiting the expression of PTEN protein. Int J Mol Med 26: 819-827, 2010.

39. Li J, Liang S, Yu H, Zhang J, Ma D and Lu X: An inhibitory effect of miR-22 on cell migration and invasion in ovarian cancer. Gynecol Oncol 119: 543-548, 2010.

40. Siemens H, Jackstadt R, Hunten S, et al: miR-34 and SNAIL form a double-negative feedback loop to regulate epithelial-mesenchymal transitions. Cell Cycle 10: 4256-4271, 2011.

41. Kim NH, Kim HS, Li XY, et al: A p53/miRNA-34 axis regulates Snail1-dependent cancer cell epithelial-mesenchymal transition. J Cell Biol 195: 417-433, 2011.

42. Preis M, Gardner TB, Gordon SR, et al: MicroRNA-10b expression correlates with response to neoadjuvant therapy and survival in pancreatic ductal adenocarcinoma. Clin Cancer Res 17: 5812-5821, 2011.
43. Bloomston M, Frankel WL, Petrocca F, et al: MicroRNA expression patterns to differentiate pancreatic adenocarcinoma from normal pancreas and chronic pancreatitis. JAMA 297: 1901-1908, 2007.

44. Teplyuk NM, Mollenhauer B, Gabriely G, et al: MicroRNAs in cerebrospinal fluid identify glioblastoma and metastatic brain cancers and reflect disease activity. Neuro Oncol 14: 689-700, 2012.

45. Huse JT, Brennan C, Hambardzumyan D, et al: The PTEN-regulating microRNA miR-26a is amplified in highgrade glioma and facilitates gliomagenesis in vivo. Genes Dev 23: 1327-1337, 2009.

46. Ciafre SA, Galardi S, Mangiola A, et al: Extensive modulation of a set of microRNAs in primary glioblastoma. Biochem Biophys Res Commun 334: 1351-1358, 2005.

47. Chisholm KM, Wan Y, Li R, Montgomery KD, Chang HY and West RB: Detection of long non-coding RNA in archival tissue: correlation with polycomb protein expression in primary and metastatic breast carcinoma. PLoS One 7: e47998, 2012.

48. Niinuma T, Suzuki H, Nojima M, et al: Upregulation of miR-196a and HOTAIR drive malignant character in gastrointestinal stromal tumors. Cancer Res 72: 1126-1136, 2012.

49. Kogo R, Shimamura T, Mimori K, et al: Long noncoding RNA HOTAIR regulates polycomb-dependent chromatin modification and is associated with poor prognosis in colorectal cancers. Cancer Res 71: 6320-6326, 2011.

50. Geng YJ, Xie SL, Li Q, Ma J and Wang GY: Large intervening non-coding RNA HOTAIR is associated with hepatocellular carcinoma progression. J Int Med Res 39: 2119-2128, 2011.

51. Yang J, Mani SA, Donaher JL, et al: Twist, a master regulator of morphogenesis, plays an essential role in tumor metastasis. Cell 117: 927-939, 2004.

52. Ma L, Reinhardt F, Pan E, et al: Therapeutic silencing of miR-10b inhibits metastasis in a mouse mammary tumor model. Nat Biotechnol 28: 341-347, 2010. 